\title{
The Fourier-Kelvin Stellar Interferometer: a low-complexity low- cost space mission for high- resolution astronomy and direct exoplanet detection
}

R. K. Barry, W. C. Danchi, L. D. Deming, L. J. Richardson, M. J. Kuchner, et al.

R. K. Barry, W. C. Danchi, L. D. Deming, L. J. Richardson, M. J. Kuchner, S. Seager, B. J. Frey, A. J. Martino, K. A. Lee, M. Zuray, J. Rajagopal, T. T. Hyde, R. Millan-Gabete, J. D. Monnier, R. J. Allen, W. A. Traub, "The FourierKelvin Stellar Interferometer: a low-complexity low-cost space mission for high-resolution astronomy and direct exoplanet detection," Proc. SPIE 6265, Space Telescopes and Instrumentation I: Optical, Infrared, and Millimeter, 62651L (6 July 2006); doi: 10.1117/12.672437

Event: SPIE Astronomical Telescopes + Instrumentation, 2006, Orlando, Florida , United States 


\title{
The Fourier-Kelvin Stellar Interferometer A Low Complexity, Low Cost Space Mission for High-resolution Astronomy and Direct Exoplanet Detection
}

\author{
R. K. Barry ${ }^{\mathrm{a}, \mathrm{b}}$, W. C. Danchi ${ }^{\mathrm{a}}$, L. D. Deming ${ }^{\mathrm{a}}$, L. J. Richardson ${ }^{\mathrm{a}, \mathrm{h}}$, M. J. Kuchner ${ }^{\mathrm{a}}$, S. Seager ${ }^{\mathrm{h}}$, B. J. Frey ${ }^{\mathrm{a}}$, \\ A. J. Martino ${ }^{a}$, K. A. Lee ${ }^{a}$, M. Zuray ${ }^{\mathrm{a}}$, J. Rajagopal ${ }^{\mathrm{a}, \mathrm{g}}$, T. T. Hyde ${ }^{\mathrm{a}}$, R. Millan-Gabet ${ }^{\mathrm{e}}$, J. D. Monnier ${ }^{\mathrm{f}}$, \\ R. J. Allen ${ }^{\mathrm{b}, \mathrm{c}}$, W. A. Traub ${ }^{\mathrm{d}}$, \\ ${ }^{a}$ NASA Goddard Space Flight Center, Greenbelt, MD 20771, USA \\ ${ }^{\mathrm{b}}$ The Johns Hopkins University, 3400 N. Charles Street Baltimore, MD 21218 \\ ${ }^{\mathrm{c}}$ Space Telescope Science Institute,3700 San Martin Dr., Baltimore, MD 21218, USA \\ ${ }^{\mathrm{d}}$ Jet Propulsion Laboratory 4800 Oak Grove Drive Pasadena, California 91109 \\ ${ }^{\mathrm{e}}$ California Institute of Technology, 1201 E. California Blvd., Pasadena, CA 91125, USA \\ fUniversity of Michigan, Astronomy Department, Ann Arbor, MI 48109, USA \\ ${ }^{g}$ University of Maryland, Astronomy Department, College Park, MD 20742, USA \\ ${ }^{\mathrm{h}}$ Carnegie Institution of Washington, 5241 Broad Branch Rd., NW, Washington, DC 20015, USA
}

\begin{abstract}
The Fourier-Kelvin Stellar Interferometer (FKSI) is a mission concept for a spacecraft-borne nulling interferometer for high-resolution astronomy and the direct detection of exoplanets and assay of their environments and atmospheres. FKSI is a high angular resolution system operating in the near to midinfrared spectral region and is a scientific and technological pathfinder to the Darwin and Terrestrial Planet Finder (TPF) missions. The instrument is configured with an optical system consisting, depending on configuration, of two $0.5-1.0 \mathrm{~m}$ telescopes on a $12.5-20 \mathrm{~m}$ boom feeding a symmetric, dual MachZehnder beam combiner. We report on progress on our nulling testbed including the design of an optical pathlength null-tracking control system and development of a testing regime for hollow-core fiber waveguides proposed for use in wavefront cleanup. We also report results of integrated simulation studies of the planet detection performance of FKSI and results from an in-depth control system and residual optical pathlength jitter analysis.
\end{abstract}

Keywords: nulling interferometer, symmetric Mach-Zehnder nuller, extrasolar planets, giant planets, planet formation, debris disk, habitable zone

\section{INTRODUCTION}

The spectroscopic resolving capability and the high spatial resolution of the FKSI instrument together with its calculated sensitivity will position it as an important facility for the study of a range of astronomical phenomena. Its science objectives are, very broadly, to directly detect and characterize the atmospheres of extrasolar giant planets (EGP), study debris disks and the evolution of protostellar and evolved stellar systems, and to facilitate the study of extra-galactic star formation regions and the extended neighborhoods of active galactic nuclei. The detection and study of EGPs will be greatly facilitated by a dedicated nulling interferometer on a satellite platform operating at infrared wavelengths. To answer key questions about these planets FKSI will be able, depending on configuration, to detect at least 25 known EGPs and make precise determination of their orbital parameters. The spatial resolution of the Fourier-Kelvin Stellar Interferometer together with the instrument's $R=25$ spectroscopic capability will allow the direct detection and analysis of photons from EGP, under certain conditions of orbital phase and angular separation. (See Danchi, et. al. 2003b.) These measurements will enable studies of the environmental conditions to which the planet is subject. They will also provide a means of measuring the composition of the planet's atmosphere. FKSI exploits the orbital dynamics of close-in planets. Using a spatial dither of the null fringe,

Space Telescopes and Instrumentation I: Optical, Infrared, and Millimeter, edited by John C. Mather, Howard A. MacEwen, Mattheus W.M. de Graauw,

Proc. of SPIE Vol. 6265, 62651L, (2006) · 0277-786X/06/\$15 · doi: 10.1117/12.672437

Proc. of SPIE Vol. 6265 62651L-1 
FKSI will be able to reconstruct the astrometric orbit of the planet and, in combination with radial velocity studies, derive the mass. In addition to mass, FKSI observes the variation in the planet's infrared spectrum as a function of phase, and facilitates characterization of the planet's thermal state and composition. In favorable cases, data obtained using FKSI could even allow scientists to make inferences about dynamical properties such as winds. This approach of exploiting the infrared brightness of close-in planets builds on the transit and radial velocity studies, which have detected most of the known extrasolar planets.

While direct detection of EGPs is a principal goal of the FKSI mission, study of stellar environments in general will also be greatly facilitated by the instrument's spatial and spectral resolving power. With FKSI, scientists may observe the formative stages of planetary systems, protoplanetary disk structure, the evolution of primordial exozodiacal dust, and debris disks about various stellar types. These observations may then be used to determine the characteristics of circumstellar material that could lead to planet formation or the evolution of the habitable zone about post-main sequence stars. These studies would facilitate refinement of the target lists and science goals of the TPF mission.

\section{MISSION CONCEPT}

Our team, lead by our Principal Investigator, Dr. W. C. Danchi, consists of scientists from a broad array of institutions and, together with engineering support from GSFC, has expended significant effort to develop a range of design options for FKSI. We have studied various beam combination techniques and array architectures in preparation for submission of FKSI as a Discovery-class mission. These design studies were conducted initially at GSFC's Instrument Synthesis and Analysis Laboratory and the Integrated Mission Design Center. These are important functions within GSFC's infrastructure used to facilitate rapid vetting of various mission and instrument design concepts. These studies were then augmented by the work of a larger, focused team of experienced scientists and engineers dedicated to the FKSI mission. The resulting design is a nulling interferometer configuration with an optical system consisting of two $0.5-1 \mathrm{~m}$ telescopes on a $12.5-20 \mathrm{~m}$ boom feeding a symmetric Mach-Zehnder beam combiner. A null tracker and hollow-core fiber for wavefront cleanup further augment the system and allow it to produce the required $.01 \%$ null of the central starlight. The flowdown of requirements from science goals to key technologies is given in figure 1.

\begin{tabular}{|c|c|c|c|}
\hline Science Goals & \multicolumn{2}{|c|}{$\begin{array}{l}\text { FKSI Requirements Flowdown } \\
\text { Measurement Capabilities Engineering Implications }\end{array}$} & Key Technologies \\
\hline $\begin{array}{l}\text { Characterize } \\
\text { Extrasolar Giant } \\
\text { Planet } \\
\text { Atmsopheres } \\
\text { Measure resonant } \\
\text { disk structures in } \\
\text { exo-zodiacal } \\
\text { debris disks to find } \\
\text { and characterize } \\
\text { extrasolar planets }\end{array}$ & \begin{tabular}{|l} 
Near-IR and Mid-IR \\
Imaging and \\
Spectroscopy \\
Spectral Range \\
$\sim 3-8 \mu \mathrm{m}$ \\
Angular Resolution \\
$\sim 41(\lambda / 5)$ mas \\
Spectra Resolving \\
Power \\
$\lambda / \Delta \lambda \sim 25$ \\
Field of View \\
$\sim 1-2$ arcsec \\
Sensitivity \\
$<2 \mu J y$ continuum \\
Observations \\
At least one target \\
field per day
\end{tabular} & $\begin{array}{l}\text { Optical System and } \\
\text { Metrology } \\
\cdot 2 \text { light collectors plus } \\
\text { nulling beam combiner } \\
\text { and spectrometer } \\
\cdot \text { Baseline } \sim 12 \mathrm{~m} \\
\text { - 0.5-1 m diameter } \\
\text { collector mirrors } \\
\text {-65 K optics } \\
\sim \lambda / 10 \text { rms at } 632 \mathrm{~nm} \\
\cdot \text {-Delay line metrology } \\
\sim 3 \mathrm{~nm} \\
\cdot \sim 15 \mathrm{~nm} \text { rms pathlength } \\
\text { control requirement } \\
\cdot \text { Sub-arcsecond relative } \\
\text { pointing } \\
\text { Detectors } \\
<10 \mathrm{e}-/ \mathrm{s} \text { dark current } \\
<10 \mathrm{e}-\text { read noise } \\
\sim 128^{\wedge} 2 \text { pixels } \\
\text { Orientation } \\
\text {-Able to view }>+/-20^{\circ} \\
\text { from ecliptic }\end{array}$ & $\begin{array}{l}\text { Detectors } \\
\text {-Very low dark current } \\
\text {-Very low read noise } \\
\text { - } 35 \text { Koperating } \\
\text { temperature desired } \\
\text { Active and Passive } \\
\text { Cooling } \\
\text {-Efficient high-capacity } \\
\text { cryocoolers } \\
\text { - 30 K cryocoolers } \\
\text {-Deployable mult-layer } \\
\text { sunshades } \\
\text { Structures and } \\
\text { mechanisms } \\
\text {-Deployable truss with } \\
\text { light collectors } \\
\text { Interferometry } \\
\text {-Cryogenic high } \\
\text { precision delay line } \\
\text {-Cryogenic optical } \\
\text { fibers for beam cleanup }\end{array}$ \\
\hline
\end{tabular}

Figure 1. FKSI requirements flowdown showing derivation of specific engineering requirements and key technologies and their relationship to mission science goals. 


\section{DYNAMIC JITTER ANALYSIS}

An in-depth control system and dynamic jitter analysis has been conducted for the FKSI mission. (See Hyde, et. al., 2004) This analysis consisted of several distinct phases. First, a structural finite element analysis was conducted using a NASTRAN model of the spacecraft bus and instrument payload systems. A normal modes analysis was performed and mode shapes and frequencies provided for the integrated model. Important nodes in the model were input degrees of freedom (DOF) at the reaction wheel assemblies (RWA) mounted to spacecraft, output DOFs at each optical element in train, and interface DOFs at the four mounting points of spacecraft to payload. The first six modes of the combined payload/spacecraft are the isolator modes and range from 0.3 to $3 \mathrm{~Hz}$ in the nominal design. Higher modes include boom-flapping modes at 5.6 and $7.3 \mathrm{~Hz}$. A mode at $25.5 \mathrm{~Hz}$ includes the motion of the combiner relative to the siderostats and strongly influences optical path delay (Figure 2).

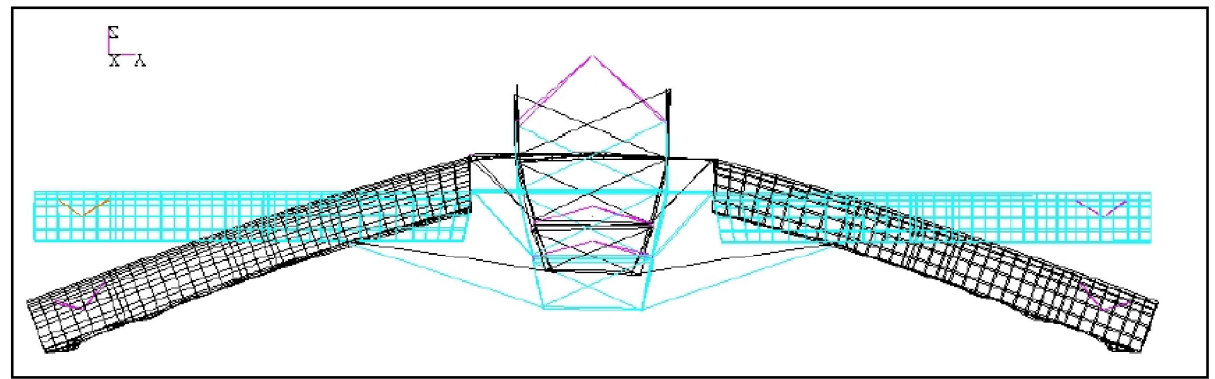

Figure 2. The FKSI NASTRAN model "wing flapping" mode.

The second phase of the analysis consisted of development of an optical model and included tracing of ray bundles from the entrance pupils above the siderostats through the optical train to the angle tracker, fringe tracker, and nuller detectors (Figure 3). This model may be described as a first-order Taylor expansion of the optical path lengths of a grid of rays traced through the system, uniformly spaced at the entrance pupil. The variables expanded upon are the rigid body DOFs for each optical component in the system. All ray tracing for the linear optical model was performed ahead of time to generate linear sensitivities that acted as transfer functions converting rigid body motions of optical components to absolute image motion at the detectors and wavefront error induced due to the misaligned components. Comparing wavefront maps generated from the sensitivities to those generated by ray tracing show less than 1 percent difference for random perturbations of one micron motions or one micro-radian tilts.

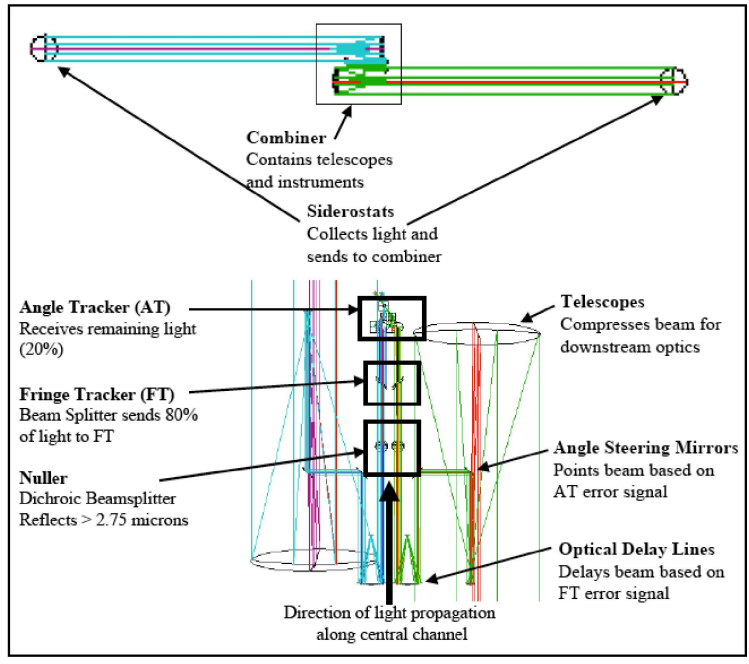

Figure 3. FKSI Optical model. 
The third modeling effort included the normal spacecraft attitude control as well as control of many optical elements in the payload. This model, carried out in MATLAB Simulink, includes the spacecraft attitude control system (ACS) as well as the two types of continuous control loops in the payload, the optical path delay (OPD) and the angle steering mirrors (ASM). The ACS uses star trackers and gyros to estimate the pointing of the spacecraft bus and controls this to about 1 arcsec RMS in roll and pitch (Figures 4 and 5). The instrument errors consist of two tilt errors and the OPD. These are sensed by the angle tracker and fringe tracker respectively and are controlled in a feedback loop to the angle steering mirrors and the optical delay line (ODL) fine stage. Sensor noise in the FT accounts for about $5 \mathrm{~nm}$ of OPD error. Actuation noise in the ODL fine stage accounts for about $5 \mathrm{~nm}$ of OPD error. The low frequency ACS control residual of about 1 arcsecond results in about $60 \mu \mathrm{m}$ of OPD, which is reduced to less than $7 \mathrm{~nm}$ by pathlength variation control. By including an unloading loop from the ODL command back to the pitch axis of the ACS, the ACS control residuals are further reduced by a factor of 100. This means that the fine stage of the ODL needs only $2 \mu \mathrm{m}$ of stroke.

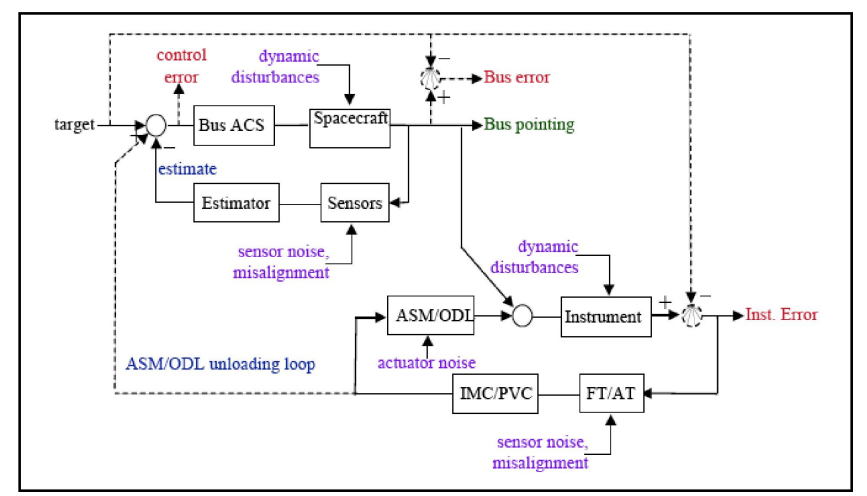

Figure 4. FKSI control system block diagram

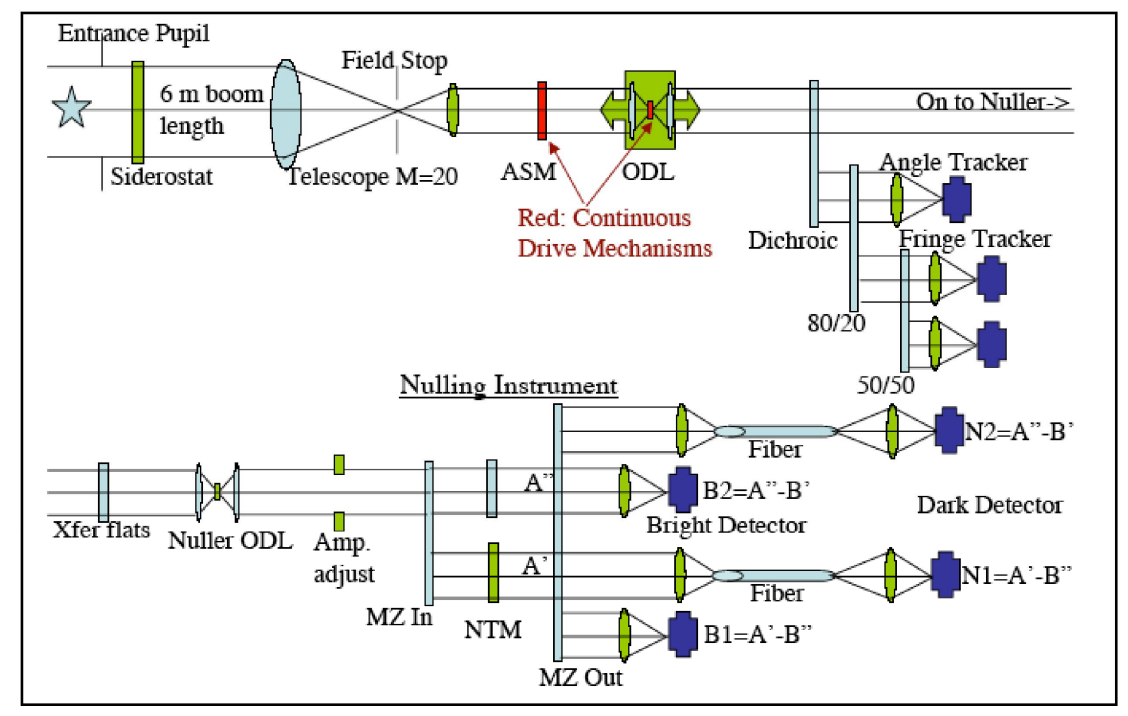

Figure 5. FKSI optical element control diagram.

A fourth element is the passive vibration isolation between the payload and the spacecraft bus. The isolator system reacts to the relative displacement between the payload and spacecraft and generates relative forces that act on the two masses. The transfer function between relative forces and relative displacements between the two masses behaves similarly to a high-pass filter. In the model of this system, the isolator 
physical parameters are mapped directly from three isolator design parameters that describe the desired isolation behavior. The baseline design has an isolation corner frequency of approximately $0.8 \mathrm{~Hz}$ with approximately $40 \%$ damping.

Finally, the results of the discipline models outlined above were integrated into a single system model. The model integration and subsequent analysis was performed in the Disturbance-Optics-Controls-Structures (DOCS) environment, a suite of tools developed at the Massachusetts Institute of Technology for performing integrated modeling, critical parameter identification, and system design (Figure 6). The influence of each design parameter and anticipated disturbance was explicitly evaluated. Critical parameters were then traded to generate a design that meets requirements optimally with respect to mission cost and risk.

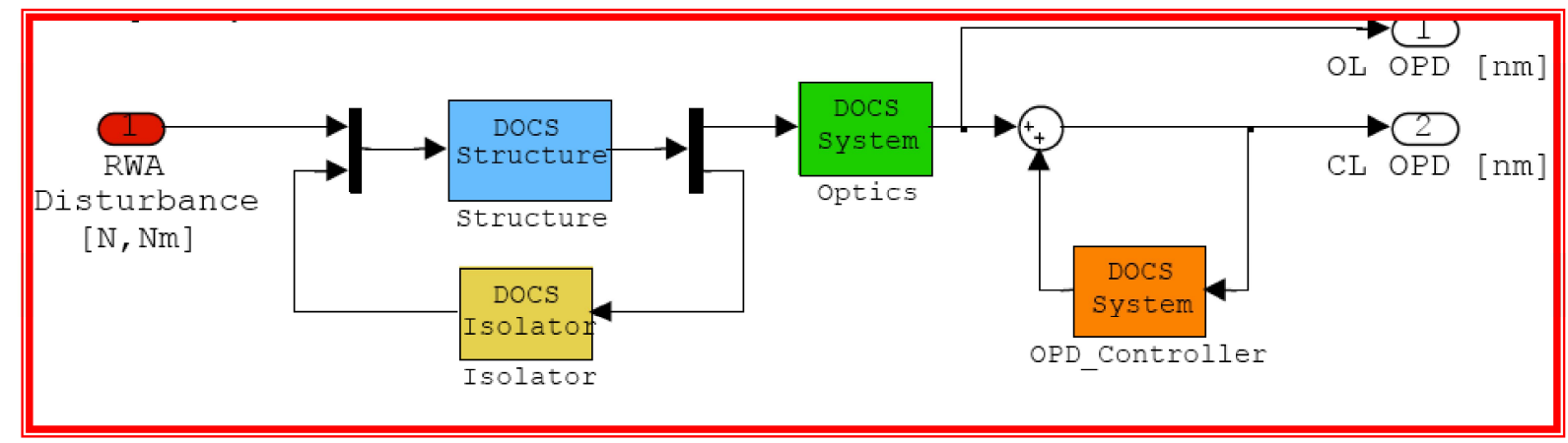

Figure 6. DOCS environment diagram for FKSI.

Null depth, a critical final output parameter of the integrated analysis, is affected by alignment, control, and dynamic stability concerns. Science drivers require a $0.01 \%$ null depth that may then be allocated across the derived null depth loss contributors. Null loss formulae subsequently yield a tolerance on parameter for each subsystem. The high-fidelity analysis allows the budgeted OPD tolerance term to be further broken down into separate component contributions, which are incoherent and are, therefore, root-sum-squared (RSS) rather than directly summed. Finally, these parameters are directly applied to FKSI testbed OPD components as design requirements. The ACS, RWA, and boom noise contributions are RSS'ed and are equated in the testbed environment to residual seismic and air turbulence perturbations.

\section{THE FKSI TESTBED}

The FKSI testbed is being built in the Horizontal Flow Facility at NASA's Goddard Space Flight Center in Greenbelt, Maryland. This testbed will allow the instrument designers to evaluate technically challenging aspects of the design. (See Barry, et. al., 2005.) In particular, the instrument's symmetric Mach-Zehnder nulling architecture will be examined together with a novel ditherless quadrature phase detector approach. Significant progress has already been made on the testbed, which has now transitioned from visible to monochromatic IR testing. The nulling architecture of the testbed consists of a symmetric Mach-Zehnder beam combiner. A laser beam is collimated by an off-axis parabola, which is then passed through a mask with two apertures. This mask simulates the beams coming from the siderostats of the proposed twoaperture version of the FKSI design. These beams then enter a pair of mirror-symmetric right angle periscopes, and are rotated $180^{\circ}$ resulting in a field reversal, which affects the incident polarization states by reversing the $\mathrm{s}$ and $\mathrm{p}$ plane reflections. The beams are then relayed through cats-eye retro-reflectors in order to control the interference of the beams in delay space without effecting polarization. After the beams are passed through the delay line, two beamsplitters split both beams, and two more beamsplitters recombine them. The beamsplitters and the fast steering mirrors are controlled by picomotors for fine adjusting. All four recombined beams are sent to four detector channels, which are used to gather both scientific data and metrology. 
After determining the cause and solution to several alignment limitations using a $632 \mathrm{~nm}$ source, we have now converted to a 1.15/3.39 $\mu \mathrm{m}$ source and have upgraded several optics. In particular, we have replaced all silver fold mirrors with uncoated gold and replaced commercial off-the-shelf (COTS) visible light beamsplitters with (50/50) IR beamsplitters. Some near-term goals include the integration of hollow, single-mode fibers for wavefront cleanup and the addition of a phase tracking control system. Phase tracking will be performed with a novel ditherless quadrature phase detector and a voice coil or piezo actuator driven in closed loop using a digital signal processing system. The phase tracking control loop will have a high bandwidth to achieve good disturbance rejection capability even at high frequency where the structural resonant modes dominate. Picomotor actuators set on four mirrors, four beamsplitters and the cats-eye phase-delays will be controlled with control system software in order to continuously track and steady the fringe during measurements. Fringe contrast will be further improved by mounting hollow fibers in the detector space to clean the wavefront of aberrations. The hollow glass waveguide (HGW) fibers chosen for the testbed have been fabricated at Rutgers University and will soon be integrated into the FKSI testbed. These HGW's were fabricated using wet-chemistry methods to first deposit a silver layer on the inside of silica glass tubing and then to form a silver halide dielectric layer over the metallic film. (See Harrington, 2001.) One advantage of these HGW fibers over the more typical solid-core polycrystalline (PC) fibers is that they can be created with a very flat spectral response in the $3-20 \mu \mathrm{m}$ spectral region. In contrast, PC fibers often suffer from mode mixing and higher loss than HGW fibers due to the extrusion pressure necessary to force the pre-form through the die. This results in the non-circularity of the core and cladding and contributes to transmission of multiple modes. HGW, in contrast, have no Fresnel loss and require no end polishing to attain single mode performance. HGW made using silica tubing such as those chosen for the FKSI testbed are very robust, and, most importantly, have been shown to have more than $20 \mathrm{~dB}$ of mode rejection between the HE11 and HE12 modes even though their bore size is significantly greater than the wavelength of light. The FKSI testbed team will be testing various lengths of 100, 150, and $180 \mu \mathrm{m} \mathrm{HGW}$ in the near future and will report our findings.

\section{HOLLOW-CORE WAVEGUIDE TESTING}

We have investigated the feasibility and efficacy of several different methods for testing the efficiency of the hollow-glass waveguides (HGWs) as modal filters. The goal of our tests is to determine the efficiency of the HGWs as modal filters over the 3-8 $\mu \mathrm{m}$ spectral region. We will quantify this efficiency by fitting a Zernike polynomial to both the measured incident and resultant wavefronts; this will allow us to determine how well specific aberrations are attenuated by the HGWs.

While the immediate rationale for conducting these tests is to find the optimal mechanism by which to clean up the FKSI testbed wavefront, we also want to characterize HGWs as modal filters for future spacebased IR interferometry missions. The most complete test regimen would therefore also include characterization over a range of cryogenic temperatures, but we only intend test them at ambient, keeping open the possibility of doing future cryo testing while developing our current test setup. These breadboard tests will also be conducted in air (i.e. not in vacuum), so we plan to construct the test setup in a Plexiglas enclosure that can be backfilled with a positive pressure of gaseous nitrogen to mitigate atmospheric water vapor and carbon dioxide. Our results will also be compared to a numerical model of expected HGW performance to verify absorption and modal filtering properties of the HGWs.

Our test setup will be located at one of the output channels of the FKSI testbed. One of the testbed flat mirrors will be replaced with a $32 \times 32$ element deformable mirror that will allow us to intentionally introduce corrugations in the incident wavefront with a resolution of $340 \mu \mathrm{m}$ and an actuator stroke of 1.5 $\mu \mathrm{m}$. This will allow us to simulate a wide variety of potential aberrations and to determine the effectiveness -- if any -- of the HGW in mitigating them. After leaving the deformable mirror, a pellicle beamsplitter will pick off a portion of the incident wavefront for detection and characterization. The remainder of the wavefront will be re-imaged onto the input end of the HGW. The output wavefront will be measured and compared to the input wavefront with as little temporal latency as possible to mitigate any non-common path environmental sources of wavefront error (e.g. air turbulence). For imaging both the incident and output wavefronts we will be using a $\mathrm{HgCdTe}$ based IR array camera with a spectral response 
that covers our entire $3-8 \mu \mathrm{m}$ bandpass. The array consists of $640 \times 48020 \mu \mathrm{m}$ pixels and will be LN2 cooled to minimize the background signal in our room temperature testbed.

After each incident and output wavefront image pair are recorded by the IR camera, we can use one of our high precision cat's eye delay lines to change the optical path difference (OPD) between the two interfering beams of the Mach-Zehnder by a well-known amount (e.g. $\lambda / 4$ ). The process is then repeated for several different OPDs as in a traditional phase shifting interferometer. The resulting images are then compiled into a data cube which contains the measured intensity $\mathrm{I}(\mathrm{x}, \mathrm{y}, \mathrm{z})$ for pixel $(\mathrm{x}, \mathrm{y})$ at OPD $\mathrm{z}$. By looking at the series of different $\lambda$ 's, one can map out and fit a polynomial function to the incident and output wavefronts to determine the efficiency of the HGWs.

\section{OPTICAL PATHLENGTH CONTROL}

Cat's Eye Delay Line (CEDL) control is accomplished in two stages, coarse and fine control. Two-tier control scheme is chosen so that the acquisition of the target fringe can be accomplished over the needed travel span by the coarse control without having to maintain the nanometer accuracy required for the eventual position stability (Figure 7). Once the coarse mechanism positions the CEDL assembly within the fine mechanism's tracking range, the coarse mechanism stops and the fine control closes the loop to achieve the nanometer position tracking stability of CEDL. The main challenge of the CEDL control lies in the fine control where desired control signal strength is close to the noise level of the controller and actuator driver.

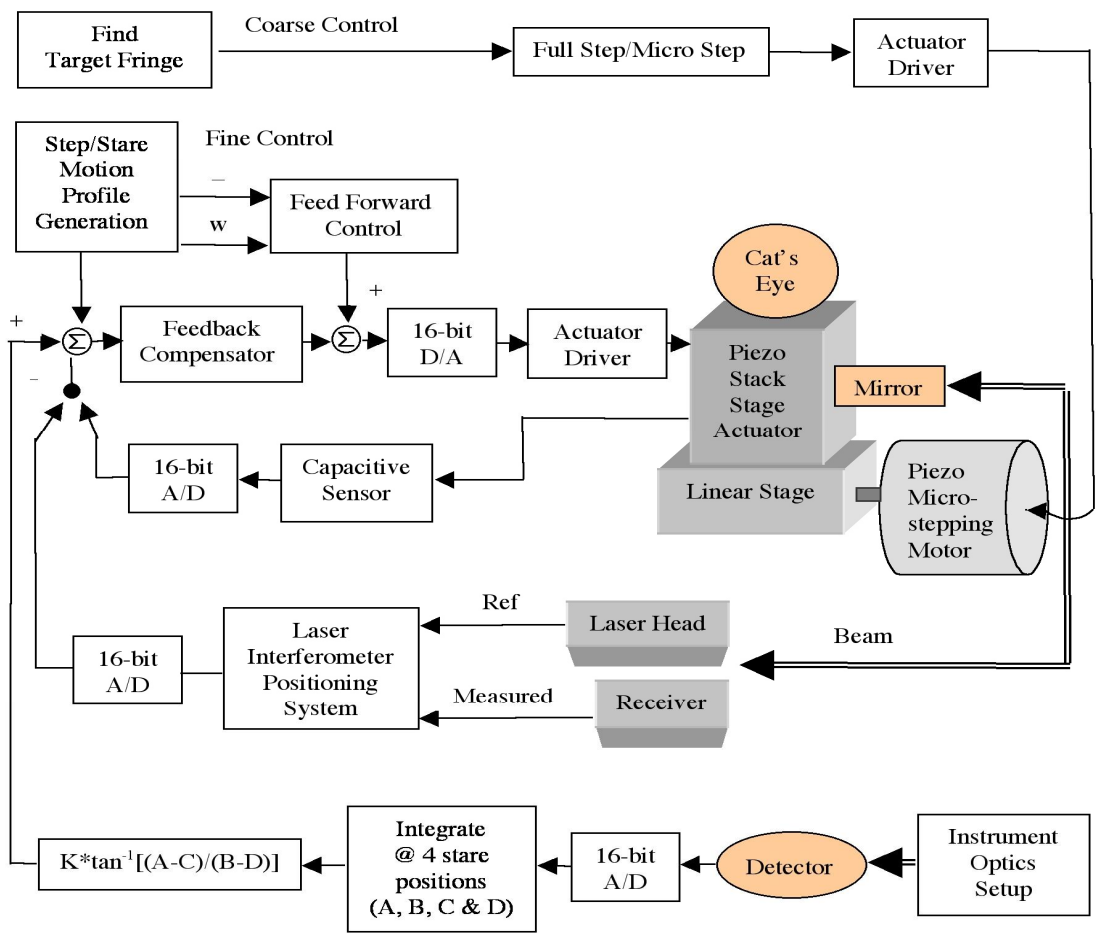

Figure 7. Simplified optical pathlength control system block diagram.

The coarse mechanism drives a linear stage that the fine control mechanism with the cat's eye and the primary mirror are mounted on. The mechanism is controlled using a direct-drive lead screw driven by a piezoelectric micro-stepping motor, has the travel range of about $12 \mathrm{~mm}$, steps in about $160 \mathrm{~nm}$ full step 
increment with micro-step capability, and employs open loop control to find the target fringe (which will be the peak white light fringe eventually). The beam wavelength for the testbed is expected to be between 3 and $8 \mu \mathrm{m}$ and the full step size of the actuator is enough to position the mechanism well within a close proximity of the target. Once the target fringe is acquired, the coarse control motion can be frozen with the piezoelectric driver turned off. A remote computer via RS-232 interface does the control of the mechanism.

The fine mechanism drives a linear stage that the cat's eye is mounted on in dithering fashion about the center of the target fringe to create changes in the beam's relative pathlength. Instead of following a typical saw-tooth slew motion, the fine mechanism is commanded to follow a step and stare motion profile while maintaining the position stability during each stare within $5 \mathrm{~nm}$. The stare time will provide a longer integration time for the detector and thus result in better signal power measurement. The $5 \mathrm{~nm}$ dynamic stability figure is from the OPD error budget allocation. The actuator that drives the linear stage is a piezoelectric stack and the required drive voltage is $100 \mathrm{~V}$ for $38 \mu \mathrm{m}$ of motion. There are four step positions, and stare time at each step position depends on the beam signal strength, fidelity of the detector signal and the detector bandwidth. Currently, the target dither frequency is around $10 \mathrm{~Hz}$ due to the bandwidth limitation of the detector.

To accomplish the required task, the fine control loop is closed as follows. A command profile is generated by combining the dithering step profile and the computed center peak of the target fringe based on the integrated detector signals at 4 stare positions. Both the feed-forward and feedback control algorithms are implemented to optimize the transient and steady state responses of the step motion. An Agilent laser interferometer system is the primary feedback sensor for position sensing. A high precision capacitive sensor is also available with an absolute position sensing capability and will be mainly used for system characterization. The capacitive sensor provides sub-nanometer resolution, and if its repeatability and nonlinearity are measured to be comparable to those of the laser system, it can be used as an alternate position sensor. The control algorithms including the command profile generation, integration of detector signals, dithering center calculation, implementation of feedback/feed-forward compensators, sensor calibration and timing sequence are accomplished in Mathworks' Simulink environment. The C-coded program is generated through Mathworks' Real-Time Workshop and is loaded onto dSpace real-time processor board that operates on TMS320C40 DSPs.

The resolution specification of Agilent laser system is $1.2 \mathrm{~nm}$ and reaches up to $12 \mathrm{kHz}$ of bandwidth. The range of actuator drive signal at the output of D-to-A interface board is from 0 to $10 \mathrm{~V}$ and a driver module delivers the gain of 10 amplification to provide 0 to $100 \mathrm{~V}$ output. DSpace system utilizes 16-bit A-to-D and D-to-A converters for its inputs and outputs, and the quantization level of the drive signal results in $0.15 \mathrm{mV}$. For $38 \mu \mathrm{m}$ range, $1.2 \mathrm{~nm}$ equates to $0.3 \mathrm{mV}$ for the servo signal. As a result, sub $\mathrm{mV}$ signal noise can impact the system performance. The estimated throughput rate of the digital control system including the input/output interface is above a couple of $\mathrm{kHz}$. The bottleneck of the controller is the detector with its maximum sample rate of $100 \mathrm{~Hz}$. Given the detector bandwidth, the present controller should be able achieve about $20 \mathrm{~Hz}$ of closed loop bandwidth.

The Dynamic jitter analysis calls for $5 \mathrm{~Hz}$ closed loop bandwidth to null the OPD error induced by the external disturbances, mainly reaction wheels. The dynamic jitter analysis, though, is based on the ditherless quadrature phase detector but the present step and stare control algorithm entails transient response, associated time delay, and possible self-induced jitter contribution in addition to external disturbances. Higher than $5 \mathrm{~Hz}$ closed loop bandwidth is needed for the present testbed to better control the transient behavior and to achieve good disturbance rejection capability.

Piezoelectric actuators are chosen for both the coarse and fine control mechanisms to achieve the high mechanical stiffness, speed and, in the case of the fine mechanism, to eventually attain high closed loop bandwidth and high disturbances rejection capability. Piezoelectric actuators have been used for space applications for the above-mentioned reasons and also for their very low power consumption. Most space applications, though, have been in ambient temperature environment and cryogenic applications do present 
challenging issues such as temperature dependence, out-gassing, insulation material, bonding material and casing. There are ongoing NASA projects that utilize piezoelectric actuators in cryogenic environment such as a tunable filter for James Webb Space Telescope and Fabry-Perot Interferometer for Next Generation Space Telescope. (See Barry, et. al., 2000.)

\section{PHASE DETECTION}

Typically, modern optical and infrared stellar interferometers measure visibilities and phases of astronomical objects by electromechanically dithering a delay line about the central fringe position thereby changing the relative pathlength light travels from each siderostat or light-gathering telescope. Measurements of power are then taken periodically as the fringe is slewed using a saw-tooth drive wave delivered to an actuator that controls physical delay. A simple phase detection algorithm is subsequently used to derive the phase from these measurements. This signed error signal, which the control system seeks to minimize in magnitude, is then conditioned into a drive signal. This drive signal is used to change the relative pathlength so that the fringe contrast - the visibility - is consistently measured. The use of this approach to sensing and control of fringe position requires considerable time per cycle resulting in a typically slow, low-bandwidth response. A more efficient possibility is to introduce additional phase shifts optically and make all measurements simultaneously to produce the error signal. This could considerably stiffen the control system response and should result in greater fringe contrast, as there will be less movement of the fringe between measurements. This should, in turn, allow improvements to the overall sensitivity of instruments using this approach, as the integration time will not be fixed by the dither frequency of electromechanical transducers - typically the principle source of bandwidth limitation in any closed loop system. Our group is developing the Ditherless Quadrature Phase Detector (DQPD) for this purpose.

The Ditherless Quadrature Phase Detector (DQPD) is being developed contemporaneously with the Fourier-Kelvin Stellar Interferometer (FKSI) testbed project and may be integrated into it depending on test results. DQPD's optical breadboard has been designed and is being built in Goddard's Horizontal Flow Facility on an optical table near the FKSI nulling testbed.

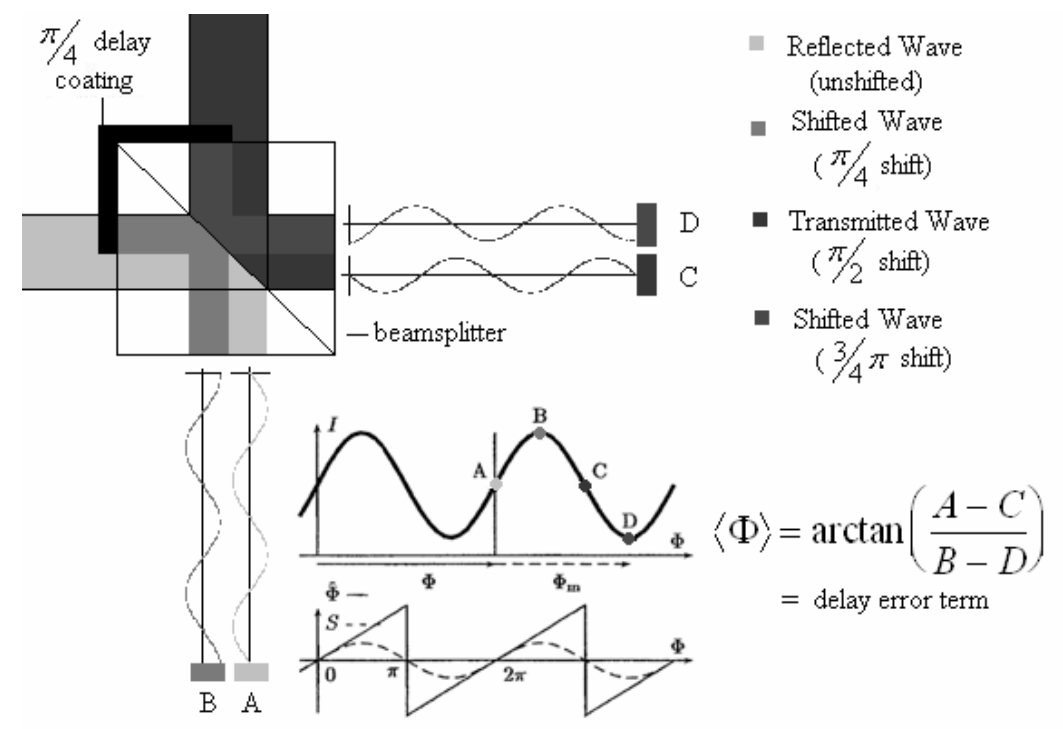

Figure 8. The Ditherless Quadrature Phase Detector for FKSI.

The principle behind DQPD may be easily understood by examining Figure 8 . This proof-of-concept design uses two beams, which are combined in a specially coated beam splitter. The coating, which delays 
half of the beam by $1 / 4 \pi$, is applied directly to the beam splitter, which is slightly wedged to reduce ghosting. The $1 / 2 \pi$ phase delay produced by transmission through the beam splitter results in the needed spread of measurements, which may be accomplished simultaneously using four detectors. The control system then rapidly calculates the required error signal as indicated in the figure. The optical breadboard is shown schematically in Figure 9.

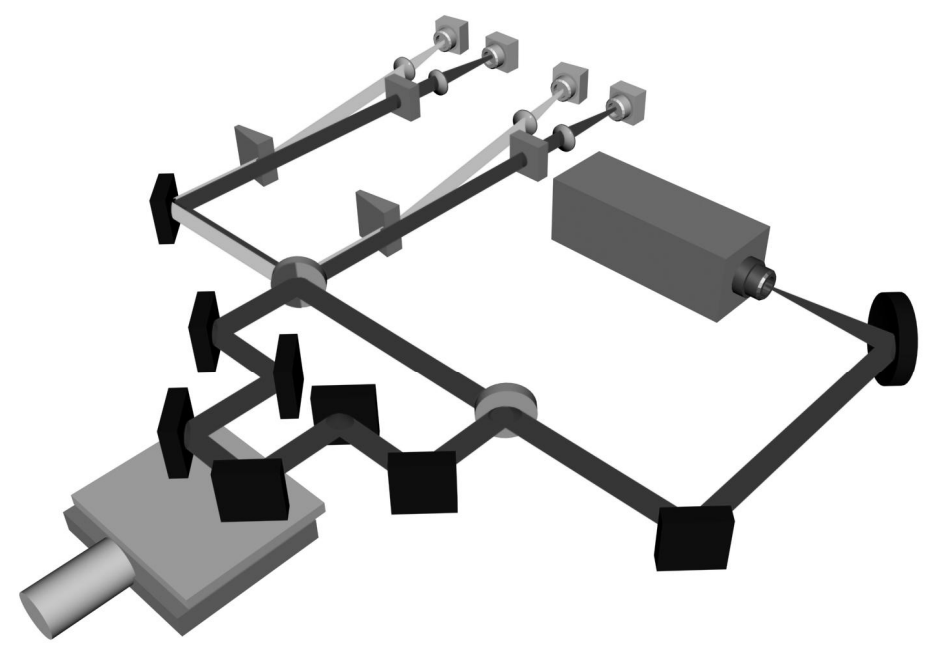

Figure 9. Schematic optical breadboard of the DQPD.

Challenges for this phase-sensor design are principally associated with our approach of using spatially separate portions of the wavefront to measure fringe power at different phases. If there are departures from ideal wavefront flatness or tilt, the error signal derived from the sensed phase will either have a systematic error term, which could be calibrated out, or will be noisy. We are now exploring designs that sample the same spatial patch of the wavefront at different polarizations or clean the wavefront and correct its tilt prior to sensing. Figure 10 shows the working breadboard of this concept.

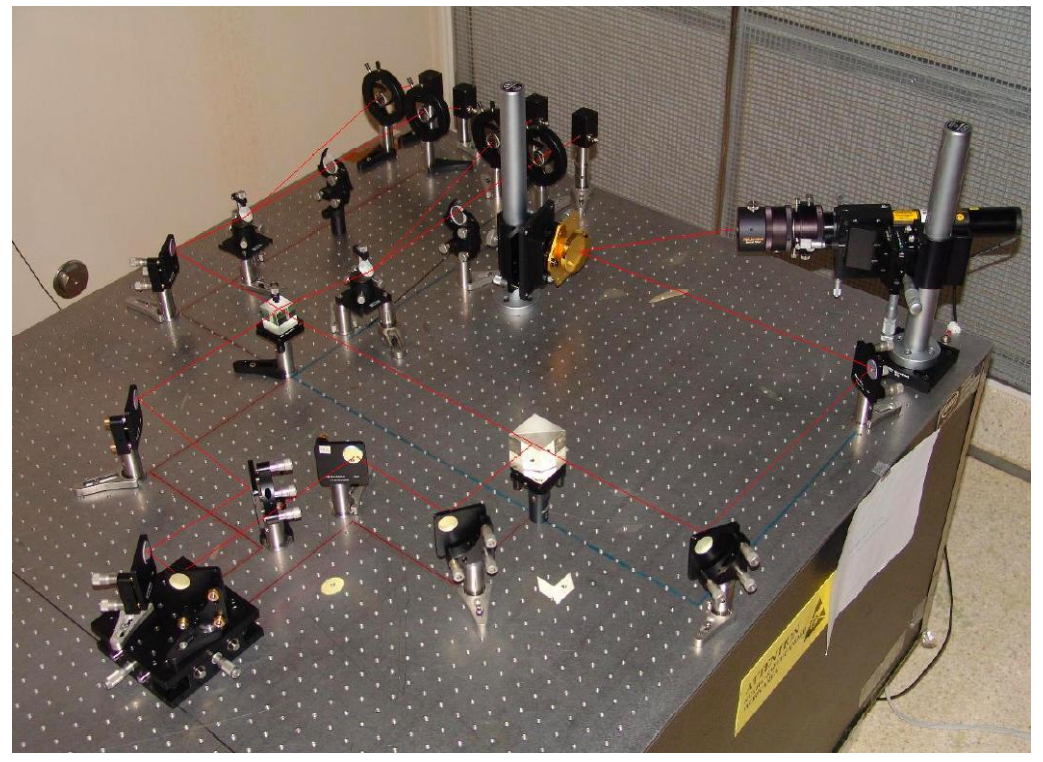

Figure 10. Photograph of functioning optical testbed for the DQPD. 


\section{THE OBSERVATORY SIMULATION CODE}

The FKSI observatory simulation code, written in IDL, is used to evaluate the detectability of known radial velocity planets. The code models the instantaneous properties of the null fringe with realistic path difference errors, and convolves the disk profile of a limb-darkened star and orbiting planet with the fringe transmission. Repeating this convolution as the interferometer rotates for a specified number of rotations at a specified rate, the code generates synthetic signals, accounting for detector noise, stellar photon noise, and noise from thermal background within the instrument and from zodiacal emission in our solar system. The code can also generate synthetic signals from hypothetical zodiacal dust in the extrasolar system using ZODIPIC, a software tool developed to compute images of exozodiacal clouds. User input to the simulation code can specify the bond albedo of the planet, and a parametric representation of how stellar irradiation is redistributed on the planetary disk. Phase-dependent variations in the planetary spectrum are also included, with orbital parameters from the Doppler data, or specified by the user. This simulation code was used to evaluate the detectability by FKSI of all know extrasolar planets as of July, 2005. (See Danchi, et. al. 2003b.) The results are given below in figure 11.

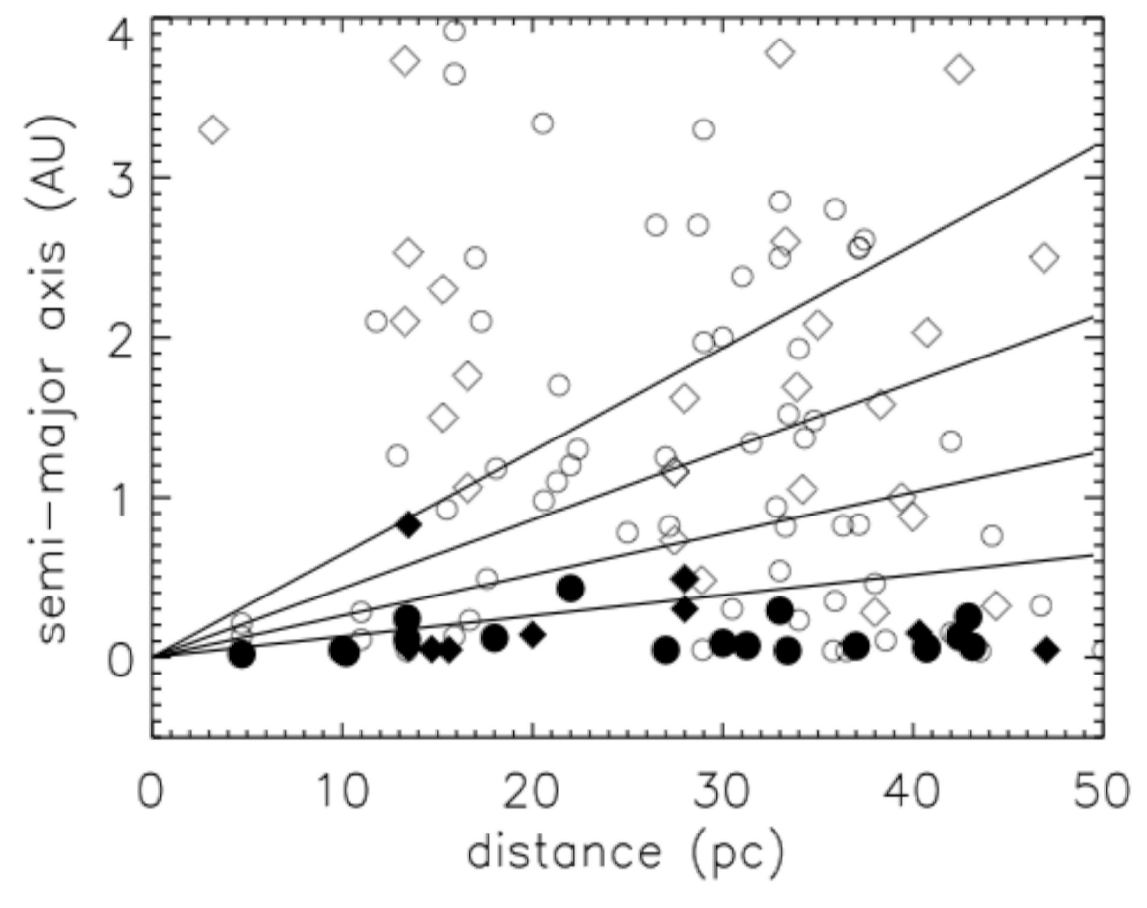

Figure 11. Plot of 140 of the known exoplanets as of July, 2005. Those detectable by FKSI within a nominal 40-degree field of regard are plotted with filled symbols. Circles indicate planets within a 20 degree field of regard, and diamonds indicate additional detectable planets if the field of regard is extended to 40 degrees. The lines correspond to the first fringe maximum for nulling interferometers of baselines 8 , 12,20 , and $40 \mathrm{~m}$ (top to bottom).

\section{ACKNOWLEDGEMENT}

This work was supported in part by NASA Goddard Space Flight Center's Internal Research and Development (IRAD) and Directors Discretionary Fund (DDF) programs. 


\section{REFERENCES}

1. Danchi, W. C., Deming, L. D., Kuchner, M. J., \& Seager, S. 2003b, ApJ 597, L57

2. T. Tupper Hyde, et. al., "Requirements Formulation and Dynamic Jitter Analysis for the Fourier-Kelvin Stellar Interferometer," Proc. SPIE, 5497, 553-564, (2004)

3. J.A. Harrington, 2001, Infrared Fiber Optics, M. Bass, J. Enoch, E. Van Stryland, and W. Wolfe, eds., (McGraw-Hill, New York)

4. R.K.Barry, et. al., "The Fourier-Kelvin Stellar Interferometer: an achievable, space-borne interferometer for the direct detection and study of extrasolar giant planets," Proc. IAU Colloquium, 200, 119-124, (2005) 5. R.K.Barry, et. al., "A Near IR Fabry-Perot Interferometer for Wide Field, Low Resolution Hyperspectral Imaging on the Next Generation Space Telescope”, in UV, Optical, and IR Space Telescopes and Instruments, James B. Breckinridge, Peter Jakobsen, Eds Proc. SPIE Vol. 4013, p. 861-871 (2000) 